\title{
ALMOST SUBNORMAL SUBGROUPS IN DIVISION RINGS WITH GENERALIZED ALGEBRAIC RATIONAL IDENTITIES
}

\author{
BUI XUAN HAI, TRUONG HUU DUNG, AND MAI HOANG BIEN
}

\begin{abstract}
In this paper we study non-central almost subnormal subgroups of the multiplicative group of a division ring satisfying a non-zero generalized rational identity. The main result generalizes Chiba's theorem on subnormal subgroups. As an application, we get a theorem on almost subnormal subgroups satisfying a generalized algebraic rational identity. The last theorem has several corollaries which generalize completely or partially some previous results.
\end{abstract}

\section{Introduction, DEFINITIONS AND MAIN RESUlts}

Let $D$ be a division ring with center $F$. We denote by $D^{*}$ the multiplicative group of $D$. In this paper, we study some algebraic valued functions in division rings. The notion of algebraic valued functions in division rings was firstly mentioned in [14] but some special cases were studied before. For instance, in [16, Kaplansky proved that if $D$ satisfies a polynomial identity, then $D$ must be centrally finite, that is, $D$ is a finite dimensional vector space over $F$. This result later was extended to division rings with generalized polynomial identities [2]. In 1966, Amitsur studied rational identities in division rings 3 and he showed that if the center of a division ring $D$ is infinite and $D$ satisfies a rational identity, then $D$ is centrally finite. In 1970, Bergman [6] extended Amitsur's work and introduced the notion of generalized rational identities. In 1996, Chiba [10] proved that if the center of a division ring $D$ is infinite and $D^{*}$ contains a non-central subnormal subgroup which satisfies a non-zero generalized rational identity, then $D$ is centrally finite. Our first aim in this paper is to carry over Chiba's result to almost subnormal subgroups. Namely, we shall prove the following theorem.

Theorem 1.1. Let $D$ be a division ring with infinite center. If $D^{*}$ contains a noncentral almost subnormal subgroup which satisfies a generalized rational identity $f=0$ for some non-zero $f(X) \in D(X)$, then $D$ is centrally finite.

Recall that in accordance with Hartley [13, a subgroup $N$ is almost subnormal in a group $G$ if there is a series of subgroups

$$
N=N_{r} \leq N_{r-1} \leq \cdots \leq N_{0}=G
$$

of $G$ such that for each $1<i \leq r$, either $N_{i}$ is normal in $N_{i-1}$ or $N_{i}$ has finite index in $N_{i-1}$. We call a series (1) an almost normal series in $G$. We say that a subgroup $N$ is almost subnormal of defect (or depth) $r$ if there is a series (1) of length $r+1$

Key words and phrases. Division ring; generalized algebraic rational identity; almost subnormal subgroup; algebraic valued function.

2010 Mathematics Subject Classification. 16R50, 16K40. 
and if no such a series of lesser length exists. The existence of almost subnormal subgroups that are not subnormal in division rings have been noted in [20].

Now, let us recall the definition of generalized rational identities. Let $D$ be a division ring with center $F$ and $X=\left\{x_{1}, \cdots, x_{m}\right\}$ be $m$ (noncommuting) indeterminates. We denote by $F\langle X\rangle$ the free $F$-algebra on $X$, by $D\langle X\rangle$ the free product of $D$ and $F\langle X\rangle$ over $F$, and by $D(X)$ the universal division ring of fractions of $D\langle X\rangle$. The existence of $D(X)$ was shown and studied deeply in [12, Chapter 7]. One calls an element $f(X) \in D(X)$ a generalized rational polynomial. If $f(X) \in D(X)$, then by [12, Theorem 7.1.2], $f(X)$ is an entry of the matrix $A^{-1}$, where $A \in M_{n}(D\langle X\rangle)$ for some positive integer $n$ such that $A$ is invertible in $M_{n}(D(X))$. If $f(X) \in F\langle X\rangle$, then we say that $f(X)$ is a polynomial in $X$ over $F$. If $f(X) \in D\langle X\rangle$, then $f(X)$ is a generalized polynomial in $X$ over $D$. If all coefficients of a generalized rational polynomial $f(X)$ are in $F$, then $f(X)$ is said to be a rational polynomial in $X$. Let $c=\left(c_{1}, \cdots, c_{m}\right) \in D^{m}$ and $\alpha_{c}: D\langle X\rangle \rightarrow D$ be the ring homomorphism defined by $\alpha\left(x_{i}\right)=c_{i}$. For any $n \in \mathbb{N}$, let $S(c, n)$ be the set of all square matrices $\left(f_{i j}(X)\right)$ of degree $n$ over $D\langle X\rangle$ such that the matrix $\left(f_{i j}(c)\right)$ is invertible in $M_{n}(D)$. Let $S(c)=\bigcup_{n \geq 1} S(c, n)$ and $E(c)$ be the subset of $D(X)$ consisting of all entries of $A^{-1}$, where $A$ ranges over $S(c)$. Then, $E(c)$ is a subring of $D(X)$ containing $D\langle X\rangle$ as a subring. Moreover, there is a ring homomorphism $\beta_{c}: E(c) \rightarrow D$ which extends $\alpha_{c}$ and every element of $E(c)$ is invertible if and only if the matrix mapped by $\beta_{c}$ is nonzero. Let $f(X) \in D(X)$ and $c \in D^{m}$. If $f(X) \in E(c)$, then we say that $f(X)$ is defined at $c$ and $\beta_{c}(c)$ is denoted by $f(c)$. For any $f(X) \in D(X)$, the set of all $c \in D^{m}$ such that $f(X)$ is defined at $c$ is called the domain of $f(X)$ and is denoted by $\operatorname{DOM}_{D}(f)$. Let $f(X) \in D(X)$ and $S \subseteq D$. If $f(c)=0$ for all $c \in S^{m} \cap \operatorname{DOM}_{D}(f)$, then we say that $f=0$ is a generalized rational identity (briefly, GRI) of $S$ or $S$ satisfies the generalized rational identity $f=0$. We refer to [12, Chapter 7] and [21, Chapter 8] for further reading on generalized rational identities.

Further, we use Theorem 1.1 to study some algebraic valued functions in division rings. In [16, Theorem 4], Kaplansky proved that a division ring whose elements all are algebraic over its center of bounded degree is centrally finite. Recently, Bell et al. considered the left algebraicity over a subfield of a division ring and they proved the analogue theorem for division rings with this property [5]. In [1, Theorem 4], it was proved that if in a division ring $D$ with center $F$ all additive commutators $x y-y x$ are algebraic over $F$ of bounded degree, then $D$ is centrally finite. An analogue result for multiplicative commutators was also obtained in 9 . Namely, $D$ is centrally finite in the case when either $x y x^{-1} y^{-1}$ are algebraic over $F$ of bounded degree for all $x, y \in D^{*}$ or $\operatorname{char}(D)=0$ and there exists a noncentral element $a$ such that $a x a^{-1} x^{-1}$ are algebraic over $F$ of bounded degree for all $x \in D$ (see [9, theorems 6, 7]). Moreover, in [1, Theorem 4] and [9, theorems $6,7]$, the bound of $[D: F]$ was also given. In the following, we give the definition of a generalized algebraic rational identity (GARI) which generalizes the notion of generalized rational identity GRI given above. As an application of Theorem 1.1 we get some theorems which generalize completely or partially results mentioned above. 
Recall that an element $\alpha \in D$ is called algebraic over $F$ if $\alpha$ is a root of non-zero polynomial over $F$, that is

$$
a_{n} \alpha^{n}+a_{n-1} \alpha^{n-1}+\cdots+a_{1} \alpha+a_{0}=0
$$

for some $n \in \mathbb{N}, a_{i} \in F$ and $a_{n} \neq 0$. Let $S$ be a subset of $D$ and $f(X)$ be a generalized rational polynomial over $D$. We say that $f$ is a generalized algebraic rational identity (briefly, GARI) of $S$ (or $S$ satisfies the GARI $f$ ) if $f(c)$ is algebraic over $F$ whenever $c=\left(c_{1}, \cdots, c_{m}\right) \in S^{m} \cap \operatorname{DOM}_{D}(f)$. A GARI $f$ of $S$ is non-trivial if there exists a division ring $D_{1}$ containing $D$ as a division subring such that the center $Z\left(D_{1}\right)$ of $D_{1}$ is exactly $F$ and there exists an element $a=\left(a_{1}, \cdots, a_{m}\right) \in$ $\operatorname{DOM}_{D_{1}}(f)$ such that $f(a)$ is not algebraic over $F$. The class of non-trivial GARIs is very big. In the next, we will show that every non-zero GRI is a non-trivial GARI. Using Theorem 1.1, we shall prove the following theorem.

Theorem 1.2. Let $D$ be a division ring with infinite center and assume that $N$ is a non-central almost subnormal subgroup of $D^{*}$. If $N$ satisfies a non-trivial GARI of bounded degree, then $D$ is centrally finite.

Theorem 1.2 has the following corollaries.

Corollary 1.3. Let $D$ be a division ring with center $F$ and assume that $N$ is a non-central almost subnormal subgroup of $D^{*}$. If all elements of $N$ are algebraic over $F$ of bounded degree, then $D$ is centrally finite.

Corollary 1.4. Let $D$ be a division ring with infinite center $F$ and $N$ is a noncentral almost subnormal subgroup of $D^{*}$. If $x y-y x$ is algebraic over $F$ of bounded degree for all $x, y \in N$, then $D$ is centrally finite.

Corollary 1.5. Let $D$ be a division ring with center $F$ and assume that $N$ is a non-central almost subnormal subgroup of $D^{*}$. If $\operatorname{char}(D)=0$ and there exists an element $a \notin F$ such that axa $a^{-1} x^{-1}$ is algebraic over $F$ of bounded degree for all $x \in N$, then $D$ is centrally finite.

Corollary 1.6. Let $D$ be a division ring with center $F$ and assume that $N$ is a non-abelian almost subnormal subgroup of $D^{*}$. If $x y x^{-1} y^{-1}$ is algebraic over $F$ of bounded degree $d$ for all $x, y \in N$, then $D$ is centrally finite.

Corollary 1.3 is a broad extension of [19, Corollary 3] and the Jacobson Theorem [15. Theorem 7]; Corollary 1.4 partially extends [1, Theorem 4]; Corollary [1.5] and Corollary [1.6 partially extend [9, Theorem 7] and [9, Theorem 6] respectively. It is unfortunate that in corollaries 1.4, 1.5 and 1.6 we are not able to give the bound for the dimension of $D$ over $F$.

\section{Proof of Theorem 1.1}

Let $D$ be a division ring with center $F$ and $\phi$ be a ring automorphism of $D$. We write $D((\lambda, \phi))$ for the ring of skew Laurent series $\sum_{i=n}^{\infty} a_{i} \lambda^{i}$, where $n \in \mathbb{Z}, a_{i} \in D$, with the multiplication defined by the twist equation $\lambda a=\phi(a) \lambda$ for every $a \in D$. If $\phi=I d_{D}$, then we write $D((\lambda))$ instead of $D\left(\left(\lambda, I d_{D}\right)\right)$. It is known that $D((\lambda, \phi))$ is a division ring (see [17, Example 1.8]). Moreover, we have the following results. 
Lemma 2.1. 7, Lemma 2.1] Let $D$ be a division ring with center $F$. Assume that $K=\{a \in D \mid \phi(a)=a\}$ is the fixed division subring of $\phi$ in $D$. If the center $k=Z(K)$ of $K$ is contained in $F$, then the center of $D((\lambda, \phi))$ is

$$
Z(D((\lambda, \phi)))=\left\{\begin{array}{cl}
k & \text { if } \phi \text { has infinite order } \\
k\left(\left(\lambda^{s}\right)\right) & \text { if } \phi \text { has a finite order } s .
\end{array}\right.
$$

In particular, the center of $D((\lambda))$ is $F((\lambda))$.

Lemma 2.2. Let $D$ be a division ring and $f(X) \in D(X)$. Then $f(X) \neq 0$ if and only if there exist a division ring $D_{1}$ containing $D$ as a division subring and an $m$-tuple $a=\left(a_{1}, \cdots, a_{m}\right) \in \operatorname{DOM}_{D_{1}}(f)$ such that $f(a) \neq 0$.

Proof. $(\Rightarrow)$ Assume that $f(X) \neq 0$. Let $F$ be the center of $D$. By Lemma 2.1 the center $F((\lambda))$ of $D((\lambda))$ is infinite. Since $f(X)$ is also a generalized rational polynomial over $D((\lambda))$, without loss of generality, we can assume that $F$ is infinite because if it is necessary, we can consider $D((\lambda))$ instead of $D$. Let $\lambda_{1}, \lambda_{2}$ be noncommuting indeterminates. Denote by $D_{1}=D\left(\lambda_{1}, \lambda_{2}\right)$ the universal division ring of fractions of $D\left\langle\lambda_{1}, \lambda_{2}\right\rangle$. Then, the center of $D_{1}$ is $F$ and $D_{1}$ is centrally infinite. It is clear that $D_{1}$ contains $D$ as a division subring and $f(X)$ is a non-zero generalized rational polynomial over $D_{1}$. Now, in view of [10, Theorem 1], there exists $a=\left(a_{1}, \cdots, a_{m}\right) \in \operatorname{DOM}_{D_{1}}(f)$ such that $f(a) \neq 0$.

$(\Leftarrow)$ This implication is trivial.

Lemma 2.3. Let $D$ be a division ring and $f(X) \in D(X)$. Assume that $f=0$ is a non-zero GRI of a subgroup $S$ of $D^{*}$. If $\left(c_{1}, \cdots, c_{m}\right) \in S^{m} \cap \mathrm{DOM}_{D}(f)$ and if we put

$$
g\left(y_{1}, \cdots, y_{m}\right)=f\left(c_{1} y_{1}, \cdots, c_{m} y_{m}\right)
$$

then $g=0$ is a non-zero GRI of $S$. As a corollary, if $f$ is a non-zero GRI of $S$, then either $S^{m} \cap \operatorname{DOM}_{D}(f)=\emptyset$ or $S$ satisfies some non-zero GRI $g$ such that $(1, \cdots, 1) \in S \cap \operatorname{DOM}_{D}(g)$.

Proof. Assume that $\left(c_{1}, \cdots, c_{m}\right) \in S^{m}$ such that $f$ is defined at $\left(c_{1}, \cdots, c_{m}\right)$. Put

$$
g\left(y_{1}, \cdots, y_{m}\right)=f\left(c_{1} y_{1}, \cdots, c_{m} y_{m}\right) .
$$

It is obvious that $g=0$ is a GRI of $S$. By Lemma 2.2, to show $g \neq 0$, it suffices to find a division ring $D_{1}$ containing $D$ and $a=\left(a_{1}, \cdots, a_{m}\right) \in D_{1}^{m}$ such that $g$ is defined at $a$ and $g(a) \neq 0$. Indeed, since $f(X) \neq 0$, by Lemma 2.2, there exist a division ring $D_{1}$ containing $D$ and $a=\left(a_{1}, \cdots, a_{m}\right) \in D_{1}^{m}$ such that $f$ is defined at $a$ and $f(a) \neq 0$. Since $c_{i}$ is invertible, $g\left(x_{1}, \cdots, x_{m}\right)$ is defined at $b$ and $g(b) \neq 0$ with $b=\left(c_{1}^{-1} a_{1}, \cdots, c_{m}^{-1} a_{m}\right)$. The proof of the lemma is complete.

The next two lemmas are due to Chiba.

Lemma 2.4. 10, Lemma 5] Let $h$ be a non-central element and let $f(X)$ be a non-zero element of $D(X)$ defined at $(1, \cdots, 1)$. Then, for $2 m$ indeterminates $y_{1}, z_{1}, y_{2}, z_{2}, \cdots, y_{m}, z_{m}$, we have

$$
\begin{gathered}
g\left(y_{1}, \cdots, y_{m}, z_{1}, \cdots, z_{m}\right)=f\left(\left[z_{1}, y_{1} h y_{1}^{-1}\right], \cdots,\left[z_{m}, y_{m} h y_{m}^{-1}\right]\right) \\
=f\left(z_{1} y_{1} h y_{1}^{-1} z_{1}^{-1} y_{1} h^{-1} y_{1}^{-1}, \cdots, z_{m} y_{m} h y_{m}^{-1} z_{m}^{-1} y_{m} h^{-1} y_{m}^{-1}\right) \in D(X)^{*} .
\end{gathered}
$$


Remark 2.5. Assume that $N$ is a non-central almost subnormal subgroup of $D^{*}$ with an almost normal series

$$
N=N_{r} \leq N_{r-1} \leq \cdots \leq N_{0}=D^{*},
$$

and $N$ satisfies a non-zero GRI. If $N_{r}$ has finite index in $N_{r-1}$, then

$$
\operatorname{Core}_{N_{r-1}}\left(N_{r}\right)=\bigcap_{x \in N_{r-1}} x N_{r} x^{-1}
$$

is the normal subgroup of finite index in $N_{r-1}$. We claim that $\operatorname{Core}_{N_{r-1}}\left(N_{r}\right)$ is non-central. Indeed, if Core ${ }_{N_{r-1}}\left(N_{r}\right)$ is central, then $a^{n} \in \operatorname{Core}_{N_{r-1}}\left(N_{r}\right)$ for any $a \in N_{r-1}$, where $n=\left[N_{r-1}:\right.$ Core $\left._{N_{r-1}}\left(N_{r}\right)\right]$. Hence, $N_{r-1}$ satisfies the identity $x^{n} y^{n} x^{-n} y^{-n}=1$. By [20, Theorem 2.2], $N_{r-1}$ is central, so is $N$, a contradiction. Thus, Core $N_{r-1}\left(N_{r}\right)$ is a non-central almost subnormal subgroup of $D^{*}$ and $N$ satisfies a non-zero GRI.

Lemma 2.6. [10, Lemma 6] Let $D$ be a centrally infinite division ring with infinite center and let $N$ be a non-central almost subnormal subgroup of $D^{*}$. For any element $f(X)$ of $D(X)$, if $f(X) \neq 0$, then $N^{m} \cap \mathrm{DOM}_{D}(f) \neq \emptyset$.

Proof. Assume that

$$
N=N_{r} \leq N_{r-1} \leq \cdots \leq N_{1} \leq N_{0}=D^{*}
$$

is an almost normal series of $N$ in $D^{*}$. In view of Remark 2.5, replacing $N_{r}$ by Core $_{N_{r-1}}\left(N_{r}\right)$ if it is necessary, we can assume that $N_{r} \unlhd N_{r-1}$. One can see that the conclusion of the lemma is obvious by applying the same arguments as in the proof of [10, Lemma 6].

Now we are ready to prove Theorem 1.1 .

Proof. Assume that $N$ is an almost subnormal subgroup of $D^{*}$ of defect $r$ with an almost normal series

$$
N=N_{r} \leq N_{r-1} \leq \cdots \leq N_{1} \leq N_{0}=D^{*},
$$

and $N$ satisfies a generalized rational identity $f=0$, where $f(X) \in D(X)^{*}$. We shall prove the statement by induction on $r$. If $r=0$, that is, $D^{*}$ satisfies a non-zero GRI, then by [10, Theorem 1], $D$ is centrally finite. Assume that the statement is true for any non-central almost subnormal subgroup $N$ of defect less than $r$. We must show that the statement holds for all non-central almost subnormal subgroups of $D^{*}$ of defect $r$. Assume that $N$ is a non-central almost subnormal subgroup of $D^{*}$ of defect $r$ such that $f=0$ is a GRI of $N$. By Remark 2.5, let

$$
N=N_{r} \leq N_{r-1} \leq \cdots \leq N_{1} \leq N_{0}=D^{*}
$$

be an almost normal series of $N$ in $D^{*}$ of defect $r$ with $N_{r} \unlhd N_{r-1}$. Deny the statement, assume that $D$ is centrally infinite. By Lemma 2.6, $f$ is defined at some $\left(c_{1}, \cdots, c_{m}\right) \in N^{m}$. In view of Lemma 2.3, we can suppose that $f$ is defined at $(1, \cdots, 1)$. Since $N_{r}$ is non-central, there exists $h \in N_{r} \backslash F$. By Lemma 2.4 we have

$$
\begin{aligned}
& g\left(y_{1}, \cdots, y_{m}, z_{1}, \cdots, z_{m}\right)=f\left(\left[z_{1}, y_{1} h y_{1}^{-1}\right], \cdots,\left[z_{m}, y_{m} h y_{m}^{-1}\right]\right) \\
= & f\left(z_{1} y_{1} h y_{1}^{-1} z_{1}^{-1} y_{1} h^{-1} y_{1}^{-1}, \cdots, z_{m} y_{m} h y_{m}^{-1} z_{m}^{-1} y_{m} h^{-1} y_{m}^{-1}\right) \neq 0 .
\end{aligned}
$$

Observe that $\left[a, b h b^{-1}\right] \in N_{r}$ for any $a, b \in N_{r-1}$, so $g=0$ is also a non-zero GRI of $N_{r-1}$. By the inductive hypothesis, $D$ is centrally finite, a contradiction. 


\section{GRI AND GARI}

We will see that the class of non-trivial GARIs is very large. In fact, we will show in this section that the class of non-trivial GARIs contains non-zero GRIs and nontrivial generalized power central rational identities (see below for the definition) in some cases. For a given positive integer $n$, let $x, y_{1}, \ldots, y_{n}$ be $n+1$ noncommuting indeterminates. Consider the following generalized rational polynomial

$$
g_{n}\left(x, y_{1}, \ldots, y_{n}\right)=\sum_{\sigma \in S_{n+1}} \operatorname{sign}(\sigma) x^{\sigma(0)} y_{1} x^{\sigma(1)} \ldots y_{n} x^{\sigma(n)},
$$

where $S_{n+1}$ is the symmetric group defined on the set $\{0,1, \ldots, n\}$.

Lemma 3.1. Let $D$ be a division ring with center $F$. For any element $a \in D$, the following statements are equivalent:

(1) The element $a$ is algebraic over $F$ of degree $\leq n$.

(2) $g_{n}\left(a, y_{1}, \ldots, y_{n}\right)=0$ is a GRI on $D$.

Proof. See [4, Corollary 2.3.8].

Lemma 3.2. Let $D$ be a division ring with center $F$. An element $\alpha=a_{1} \lambda+a_{2} \lambda^{2}+$ $\cdots$ in $D((\lambda))$ is algebraic over $F$ if and only if $\alpha=0$.

Proof. Suppose that $\alpha \neq 0$ and $g(x)=t_{0}+t_{1} x+\cdots+t_{n} x^{n} \in F[x]$ is the minimal polynomial of $\alpha$ over $F$. Then, from the equality

$$
0=t_{0}+t_{1}\left(a_{1} \lambda+a_{2} \lambda^{2}+\cdots\right)+\cdots+t_{n}\left(a_{1} \lambda+a_{2} \lambda^{2}+\cdots\right)^{n}
$$

one has $t_{0}=0$, that is impossible.

For a division ring $D$ with center $F$, let us consider a countable set of indeterminates $\left\{\lambda_{i} \mid i \in \mathbb{Z}\right\}$ and a family of division rings which is constructed by setting

$$
\begin{gathered}
D_{0}=D\left(\left(\lambda_{0}\right)\right), D_{1}=D_{0}\left(\left(\lambda_{1}\right)\right), \\
D_{-1}=D_{1}\left(\left(\lambda_{-1}\right)\right), D_{2}=D_{-1}\left(\left(\lambda_{2}\right)\right),
\end{gathered}
$$

for any $n>1$,

$$
D_{-n}=D_{n}\left(\left(\lambda_{-n}\right)\right), D_{n+1}=D_{-n}\left(\left(\lambda_{n+1}\right)\right) .
$$

Clearly, $D_{\infty}=\bigcup_{n=-\infty}^{+\infty} D_{n}$ is a division ring. By Lemma 2.1, it is not hard to prove by induction on $n \geq 0$ that the center of $D_{0}$ is $F_{0}=F\left(\left(\lambda_{0}\right)\right)$, the center of $D_{n+1}$ is $F_{n+1}=F_{-n}\left(\left(\lambda_{n+1}\right)\right)$ and the center of $D_{-n}$ is $F_{-(n+1)}=F_{n+1}\left(\left(\lambda_{-(n+1)}\right)\right)$. In particular, $F$ is contained in $Z\left(D_{\infty}\right)$. Consider the map $\phi: D_{\infty} \longrightarrow D_{\infty}$ which is defined by $\phi(a)=a$ for any $a \in D$ and $\phi\left(\lambda_{i}\right)=\lambda_{i+1}$ for any $i \in \mathbb{Z}$ is an automorphism of $D_{\infty}$.

Proposition 3.3. The center of $D_{\infty}((\lambda, \phi))$ is $F$.

Proof. We note that $D$ is the fixed division ring of $\phi$ in $D_{\infty}$. Since $F$ is contained in the center of $D_{\infty}$, the automorphism $\phi$ has infinite order. By Lemma 2.1. $Z\left(D_{\infty}((\lambda, \phi))\right)=F$.

Theorem 3.4. Let $D$ be a division ring with center $F$ and $S$ be a subset of $D$. Assume that $f(X) \in D(X) \backslash D$ is a GARI of $S$. If $f(c) \in F$ for some $c=\left(c_{1}, \cdots, c_{m}\right) \in S^{m}$, then $f$ is a non-trivial GARI. 
Proof. By the definition of non-trivial GARIs, we must find a division ring $L$ with center $F$ containing $D$ as a division subring and an element $a=\left(a_{1}, \cdots, a_{m}\right)$ in $L^{m}$ such that $f(a)$ is not algebraic over $F$. Let $K=D(Y)$, where $Y=\left\{y_{1}, \cdots, y_{m}\right\}$ and $L=K_{\infty}((\lambda, \phi))$. By Proposition 3.3. $Z(L)=Z(K)=Z(D)=F$. Consider the division subring $K((\lambda))$ of $L$. By Lemma 2.1, $F((\lambda))$ is the center of $K((\lambda))$. In view of [11, Lemma 7],

$$
f(c+\lambda Y)=f\left(c_{1}+y_{1} \lambda, \cdots, c_{m}+y_{m} \lambda\right)=f(c)+\sum_{j=1}^{\infty} f_{j}(Y) \lambda^{j},
$$

where $f_{j}(Y)$ are generalized polynomials over $D$ and there is $j_{0}$ such that $f_{j_{0}}(Y) \neq$ 0 . Since $f(c) \in F$, if $f(c+\lambda Y)$ is algebraic over $F$, then $\sum_{j=1}^{\infty} f_{j}(Y) \lambda^{j}$ is algebraic over $F$ too. By Lemma 3.2, $f_{j}(Y)=0$ for every $j \geq 1$. In particular, we have $f_{j_{0}}(Y)=0$, a contradiction. Thus, $f(c+\lambda Y)$ is not algebraic over $F$. Therefore, $f$ is not a GARI of $L$.

Recall that for a division ring $D$ with center $F$, an element $f(X) \in D(X)$ is called a generalized power central rational identity (shortly, GPCRI) of a subset $S$ of $D$ if $f(X)$ satisfies the following condition: if $c=\left(c_{1}, \cdots, c_{m}\right) \in S^{m} \cap \mathrm{DOM}_{D}(f)$, then there exists a positive integer $p_{c}$ (depending on $c$ ) such that $f(c)^{p_{c}} \in F$ (see [1]). Moreover, if $f(X)^{p} \in D(X) \backslash F$ for any positive integer $p$, then we say that $S$ satisfies a non-trivial GPCRI $f(X)$. It is obvious that if $f(X)$ is a GPCRI of some subset $S$, then $f(X)$ is a GARI of $S$. In the following result, we will show that every non-trivial GPCRI is a non-trivial GARI.

Corollary 3.5. Let $D$ be a division ring with center $F$ and $f(X) \in D(X)$. Assume that $S$ is a subset of $D$ such that $S^{m} \cap \operatorname{DOM}_{D}(f) \neq \emptyset$. If $f(X)$ is a non-trivial GPCRI of $S$, then $f(X)$ is a non-trivial GARI of $S$.

Proof. Assume that $f(X)$ is a non-trivial GPCRI of $S$. Then, it is obvious that $f(X)$ is a GARI of $S$. Now we will show that $f(X)$ is a non-trivial GARI of $S$. It suffices to prove that $g(X)=f(X)^{p}$ is a non-trivial GARI of $S$ for some positive integer $p$. Indeed, since $S^{m} \cap \mathrm{DOM}_{D}(f) \neq \emptyset$, let $c=\left(c_{1}, \cdots, c_{m}\right) \in S^{m} \cap \mathrm{DOM}_{D}(f)$. Then, there exists $p_{c}>0$ such that $f(c)^{p_{c}} \in F$. Put $g(X)=f(X)^{p_{c}}$. Then it is obvious that $g(X)$ is a GARI of $S$. Since $g(c)=f(c)^{p_{c}} \in F$ which implies that $g(X)$ is a non-trivial GARI of $S$ by Theorem 3.4.

Let $D$ be a division ring with center $F$ and $S$ be a subset of $D^{*}$. We say that $S$ satisfies a non-trivial GARI of bounded degree if there exists a non-trivial GARI $f(X)$ of $S$ over $D$ such that for all $c=\left(c_{1}, \ldots, c_{m}\right) \in S^{m} \cap \operatorname{DOM}_{D}(f)$, the element $f(c)$ are algebraic over $F$ of bounded degree.

Notice that if $f=0$ is a GRI of a subset $S$ of a division ring $D$, then obviously $f(X)$ is a GARI of $S$. However, if in addition, $f(X)$ is non-zero, then $f(X)$ may not be a non-trivial GARI. For example, let

$$
D=\mathbb{R} \oplus \mathbb{R} i \oplus \mathbb{R} j \oplus \mathbb{R} k
$$

be a real quaternion algebra, $S=\{1\}$ and $f(X)=(X-1) i(X-1)^{-1}$. Then $S^{1} \cap \operatorname{DOM}_{D}(f)=\emptyset$ which implies that $f=0$ is a non-zero GRI of $S$. Moreover, since $f(X)^{2}=(X-1) i^{2}(X-1)^{-1}=-1, f(X)$ is a GARI (of degree 2) of any division ring $D_{1}$ containing $D$. Hence, $f(X)$ is a trivial GARI of $S$. The following result shows that if $f(X)$ is defined at least at an $m$-tuple in $S^{m}$, then $f(X)$ is a non-trivial GARI of $S$. 
Corollary 3.6. Let $D$ be a division ring with center $F$ and $f(X) \in D(X)$. Assume that $S$ is a subset of $D$ such that $S^{m} \cap \mathrm{DOM}_{D}(f) \neq \emptyset$. If $f=0$ is a non-zero GRI of $S$, then $f(X)$ is a non-trivial GARI of $S$ of degree 1 .

Proof. Assume that $f=0$ is a non-zero GRI of $S$. Then $f(X)$ is a GARI of $S$ of degree 1 . Now we must show $f(X)$ is non-trivial GARI of $S$. Indeed, if $f(X) \in D$, then $f(X)=0$ that is impossible since $f=0$ is a non-zero GRI of $S$. Hence $f(X) \notin D$. By the hypothesis, there exists $c=\left(c_{1}, \ldots, c_{m}\right) \in S^{m} \cap \operatorname{DOM}_{D}(f)$ such that $f(c)=0$. In view of Theorem 3.4 it follows that $f(X)$ is a non-trivial GARI of $S$.

Remark. In view of Corollary 3.6, one can see that Theorem 1.2 generalizes [10, Theorem 1]. Note that the last theorem is a generalization of a theorem of MakarLimanov [18, Theorem].

\section{Proof of Theorem 1.2}

Proof. Assume that $N$ satisfies the non-trivial GARI $f(X)$ of bounded degree $k$. Let $Y=\left\{y_{1}, \cdots, y_{k}\right\}$ and $x$ be $k+1$ indeterminates. Consider

$$
g_{k}(x, Y)=\sum_{\sigma \in S_{k+1}} \operatorname{sign}(\sigma) \cdot x^{\sigma(0)} y_{1} x^{\sigma(1)} \ldots y_{k} x^{\sigma(k)}
$$

as in Lemma 3.1, and put

$$
w(X, Y)=g_{k}(f(X), Y) .
$$

Assume that $c=\left(c_{1}, c_{2}, \cdots, c_{m}\right) \in N^{m} \cap \operatorname{DOM}_{D}(f)$. Since $f(c)$ is algebraic over $F$ of degree $\leq k$, by Lemma 3.1 .

$$
w(c, r)=0
$$

for every $r=\left(r_{1}, \cdots, r_{k}\right) \in D^{k}$. In particular, $w=0$ is a GRI of $N$. Because $f$ is a non-trivial GARI, there exist a division ring $D_{1}$ with center $F$ containing $D$ as a division subring and an element $a=\left(a_{1}, \cdots, a_{m}\right) \in \operatorname{DOM}_{D_{1}}(f)$ such that $f(a)$ is not algebraic over $F$. By Lemma 3.1, $w(X, Y) \neq 0$, and consequently, $w=0$ is a non-zero GRI of $N$. Now in view of Theorem 1.1, $D$ is centrally finite, and the proof of Theorem 1.2 is now complete.

Remark. The non-triviality of $f$ in Theorem 1.2 is essential. For instance, let $D$ be a centrally infinite division ring, and assume that $a \in D^{*}$ is an algebraic element of degree $d$ over the center $F$ of $D$. Then, for any $b \in D^{*}$, the element $b a b^{-1}$ is always algebraic over $F$ of degree $\leq d$. This means that $f(x)=x a x^{-1}$ is a GARI of $D^{*}$ while $D$ is centrally infinite.

\section{Proofs of COROLlaries}

\subsection{Proof of Corollary 1.3.}

Proof. If the center $F$ is finite, then every element of $N$ is torsion. By [20, Proposition 4.4] $N$ is central which contradicts the hypothesis. Hence, $F$ is infinite. By Theorem [1.2, $D$ is centrally finite.

\subsection{Proof of Corollary 1.4.}

Proof. The corollary is followed directly from Theorem 1.2 , 


\subsection{Proof of Corollary 1.5.}

Proof. Put $w(x)=a x a^{-1} x^{-1}$. Then $w(x)$ is a GARI of $N$ of bounded degree. Using Theorem 3.4 $w(x)$ is a non-trivial GARI of $N$ because $w(1)=1 \in F$. Since $F$ is infinite, by Theorem $1.2, D$ is centrally finite.

\subsection{Proof of Corollary 1.6.}

Proof. Put $w(x, y)=x y x^{-1} y^{-1}$. Then $w(x)$ is a GARI of $N$ of bounded degree $d$. In view of Theorem 3.4. $w$ is a non-trivial GARI of $N$ because $w(1,1)=1 \in F$. By Theorem 1.2, it suffices to show that $F$ is infinite. Indeed, assume that $F$ is finite. Then, for any $a, b \in N$, the subfield $F\left(a b a^{-1} b^{-1}\right)$ of $D$ generated by $a b a^{-1} b^{-1}$ over $F$ is finite which implies that $a b a^{-1} b^{-1}$ is torsion of order $n \leq|F|^{d}-1$. Therefore, $N$ satisfies a generalized group identity $w(x, y)^{n}=1$. If there exist $a, b \in N$ such that $a b a^{-1} b^{-1}=w(a, b) \notin F$, then there exists a division subring $D_{1}$ of $D$ with center $F_{1}$ such that $D_{1}$ is centrally finite and $a b a^{-1} b^{-1} \in D_{1} \backslash F_{1}$ ([8, Proposition 2.1]). Since $N$ is almost subnormal in $D^{*}$, there exist a series of subgroups

$$
N=N_{r} \leq N_{r-1} \leq \cdots \leq N_{1}=D^{*}
$$

such that for any $1 \leq i<r$, either $\left[N_{i}: N_{i+1}\right]<\infty$ or $N_{i+1}$ is normal in $N_{i}$. Putting $H_{i}=D_{1} \cap N_{i}$, we obtain the following series of subgroups

$$
H_{r} \leq H_{r-1} \leq \cdots \leq H_{1}=D_{1}^{*},
$$

where for any $1 \leq i<r$, either $\left[H_{i}: H_{i+1}\right]<\infty$ or $H_{i+1}$ is normal in $H_{i}$. Therefore $H=H_{r}$ is an almost subnormal subgroup of $D_{1}^{*}$ and $a b a^{-1} b^{-1} \in H$. Observe that $F_{1}$ is infinite and since $H \subseteq N$ satisfies the group identity $w(x, y)^{n}=1$, by 20, Theorem 2.2], $H \subseteq F_{1}$. In particular, $a b a^{-1} b^{-1} \in F_{1}$, a contradiction. Hence, $a b a^{-1} b^{-1} \in F$ for any $a, b \in N$. Since $N$ is non-abelian, there exist $a, b \in N$ such that $a b a^{-1} b^{-1}=\alpha \in F \backslash\{1,0\}$. Let $n$ be the order of $\alpha$. Consider the division subring $F(a, b)$ of $D$ generated by $a, b$ over $F$. Then, we also have $a b^{n} a^{-1}=$ $(\alpha b)^{n}=b^{n}$ because $a b a^{-1}=\alpha b$, hence $b^{n} \in Z(F(a, b))$. Similarly, we also have $a^{n} \in Z(F(a, b))$. If $a^{n}$ and $b^{n}$ are algebraic over $F$, then of course $a$ and $b$ are algebraic over $F$ too. In this case, it is easy to check that $F(a, b)=F[a, b]$ is finite, hence $F(a, b)$ is commutative that is impossible because $a b \neq b a$. Therefore, $a^{n}$ or $b^{n}$ is not algebraic over $F$. As a result, $Z(F(a, b))$ is infinite. By repeating the arguments in the first part with $F(a, b)$ instead of $D_{1}$, we have $F(a, b) \cap N$ is abelian. Again, this is impossible in view of inequality $a b \neq b a$. Thus, $F$ is infinite and the proof of the corollary is now complete.

\section{Acknowledgments}

This work is funded by Vietnam National Foundation for Science and Technology Development (NAFOSTED) under Grant No. 101.04-2016.18.

\section{REFERENCES}

[1] S. Akbari, M. Arian-Nejad and M. L. Mehrabadi, On Additive Commutator Groups in Division Rings, Result. Math. 33 (1998) 9-21.

[2] S. A. Amitsur, Generalized polynomial identities and pivotal monomial, Trans. Amer. Math. Soc. 114 (1965), 210-226.

[3] S. A. Amitsur, Rational identities and applications to algebra and geometry, J. Algebra 3 (1966), 304-359.

[4] K. I. Beidar, W. S. Martindale 3rd and A. V. Mikhalev, Rings with Generalized Identities, Marcel Dekker, Inc., New York-Basel-Hong Kong, 1996. 
[5] P. J. Bell, V. Drensky, Y. Sharifi, Shirshov's theorem and division rings that are left algebraic over a subfield, J. Pure Appl. Algebra 217 (2013), no. 9, 1605-1610.

[6] G. M. Bergman, Skew field of noncommutative rational functions, after Amitsur, Sem. Schutzenberger-Lentin-Nivat 1969/70 No. 16 (Paris 1970).

[7] M. H. Bien, On normal subgroups of $D^{*}$ whose elements are periodic modulo the center of $D^{*}$ of bounded order, Int. Electron. J. Algebra 16 (2014), 66-71.

[8] M. H. Bien, D. H. Dung, On normal subgroups of division rings which are radical over a proper division subring, Studia Sci. Math. Hungar. 51 (2014), no. 2, 231-242.

[9] M. A. Chebotar, Yuen Fong and Pjek-Hwee Lee, On division rings with algebraic commutators of bounded degree, Manuscripta Math. 113 (2004), 153-164.

[10] K. Chiba, Generalized rational identities of subnormal subgroups of skew fields, Proc. Amer. Math. Soc. 124(6) (1996), 1649-1653.

[11] K. Chiba, Skew fields with a nontrivial generalised power central rational identity, Bull. Austral. Math. Soc. 49 (1994), no. 1, 85-90.

[12] P. M. Cohn, Free rings and their relations, Academic Press, New York and London, 1971.

[13] B. Hartley, Free groups in normal subgroups of unit groups and arithmetic groups, Contemp. Math. 93 (1989), 173-177.

[14] I. N. Herstein, C. Procesi and M. Schacher, Algebraic valued functions on noncommutative rings, J. Algebra. 36 (1975), 128-150.

[15] N. Jacobson, Structure theory for algebraic algebras of bounded degree, Ann. of Math. 46, (1945), 695-707.

[16] I. Kaplansky, Rings with a polynomial identity, Bull. Amer. Math. Soc. 54 (1948). 575-580.

[17] T. Y. Lam, A First Course in Noncommutative Rings, in: Grad. Texts in Math., Vol. 131, Springer-Verlag, Berlin, 1991.

[18] L. Makar-Limanov, On subnormal subgroups of skew fields, J. Algebra 114 (1988), no. 2, 261-267.

[19] M. Mahdavi-Hezavehi, S. Akbari-Feyzaabaadi, M. Mehraabaadi and H. Hajie-Abolhassan, On derived groups of division rings II, Comm. Algebra 23 (1995), no. 8, 2881-2887.

[20] N. K. Ngoc, M. H. Bien and B. X. Hai, Free subgroups in almost subnormal subgroups of general skew linear groups, Algebra i Analiz, 28:5 (2016), 220-235.

[21] L. H. Rowen, Polynomial identities in ring theory, Academic Press, Inc., New York, 1980.

Faculty of Mathematics and Computer Science, VNuHCM-University of Science, 227 NGuYen Van Cu Str., Dist. 5, HCM-City, Vietnam

E-mail address: bxhai@hcmus.edu.vn

Faculty of Mathematics and Computer Science, VNUHCM-University of Science, 227 NGuyen Van Cu Str., Dist. 5, HCM-City, Vietnam

E-mail address: thdung@dnpu.edu.vn

Faculty of Mathematics and Computer Science, VNUHCM-University of Science, 227 Nguyen Van Cu Str., Dist. 5, HCM-City, Vietnam

E-mail address: mhbien@hcmus.edu.vn 